\title{
Segmentation of Planar Curves Using Local and Global Behaviour Analysis
}

\author{
L.D. Cai, J. Porrill, S.B. Pollard, J.E.W. Mayhew and J.P. Frisby \\ AI Vision Research Unit, University of Sheffield \\ Western Bank, Sheffield S10 2TN, UK
}

\begin{abstract}
In this paper a planar curve segmentation method based on the analysis of curvature and mean polygonal area is proposed. A rough segmentation of the curve is first produced by a high/low curvature classifier and then refined by a high/low/zero classifier and $a$ knot/straight/convex/concave shape identifier so as to obtain a set of critical knots and shape descriptors for all segments. A straight line or conic approximation is then applied to each segment to generate a symbolic representation of the curve.
\end{abstract}

\section{Introduction}

The TINA vision system [1] has so far been restricted to the use of lines and circles as 3D primitives. The descriptive base is now to be extended to include line, conic, and various forms of generic (e.g. spline) curve descriptors [2]. The general philosophy is to fit descriptions in the left image, then use disparity data from the right image to project these curves into space. This approach will be much more robust [3] than direct fitting in 3D space.

Curve segmentation is an under-conditioned curve approximation problem [4]. Feasible solutions can be obtained, for example, by imposing some constraints on the fitting basis functions, that is, partitioning curves into pieces using some easily described primitives. There are two basic ways to do so. The first is top down, hierarchically fitting the repertoire of primitives and inserting segmentation points where the fit breaks down. Examples of this approach are recursive fittings $[5,6]$. The second approach is bottom up, attempting to locate generic segmentation points, such as corners and smooth joins, that will be appropriate over a range of fitting methods in the later stage. This approach yields a preliminary symbolic description of the curve as a set of knots joined by arcs with descriptions such as straight/concave/convex etc.. Both approaches have their advantages and are used in the TINA system. This paper describes the second, bottom up, approach.

Note that many manufactured parts are formed from planar or quadric surfaces. The edges detected in the intensity images are therefore straight lines or conic

This research was funded by a grant of the Voila vision research pilot project (Esprit project P2502). curves and piecewise approximation by such curves is appropriate. Even outside this restricted domain segmentation into straight lines and conics gives sensible results (conic splines are a commonly used representation of general curves in computer graphics). Hence the choice of critical knots for partitioning is related to the kind of fitting basis functions to be applied.

As the knots of interest in our case are corners and various types of smooth join, and these last are, as Asada and Brady [7] point out, "hard to detect", this paper tries to segment planar curves by making an analysis of the local and global behaviour of curves with the intention of setting up appropriate thresholds to locate critical knots and describe the shape of curve segments.

\section{Local behaviour of planar curves}

Local behaviour will be analysed on the basis of the curvature near the critical knots of planar curves. As an intrinsic property of curves, curvature is invariant to rotation and translation operations, and is widely used as an indicator of corners, smooth joins and curve shapes.

One reason for a local behaviour analysis is the corner-rounding effects due to operations such as data smoothing and finite differencing [8]. According to the combination of curvature signs $(+, 0,-)$, there are six types of corner as shown in Figure 1.

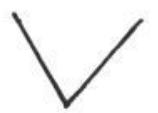

$0 / 0$

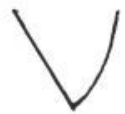

$0 /+$

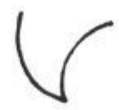

$+1-$

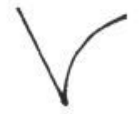

$0 /-$

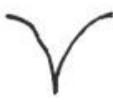

$-1-$
Figure 1. Six types of comer created by lines and arcs.

Going along a curve, sharp orientation changes of the tangent of the curve occur at corners, and produce high curvature there. Therefore, corners are located as the high curvature knots. However, a high curvature threshold is not sufficient to locate them due to corner- 
rounding effects. Even when a corner is created by the intersection of two straight lines (i.e., a corner of $0 / 0$ type), curvature magnitudes at the neighbouring points of the corner will be raised. As the corner rounding effect from data smoothing is a well-known fact, we discuss only the effect from finite differencing below.

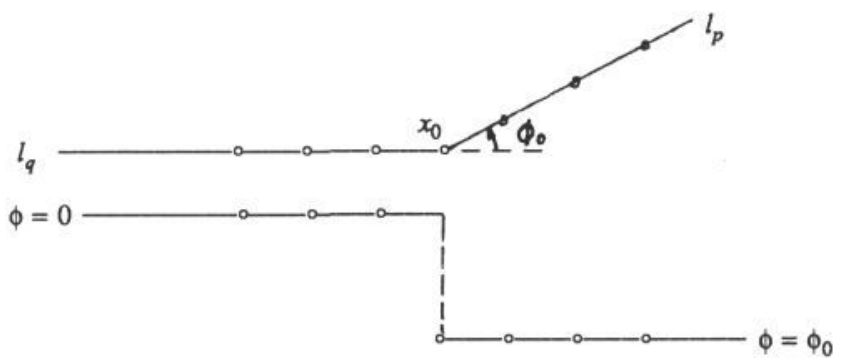

$$
\begin{array}{lllllll}
-3 & -2 & -1 & 0 & 1 & 2 & 3
\end{array}
$$

Figure 2. A comer $x_{0}$ as the intersection of two lines $l_{q}$ and $l_{p}$ and the tangent orientation $\phi$ near $x_{0}$.

Let $p$ and $q$ be the slopes of $l_{p}$ and $l_{q}$ respectively in Figure 2. When finite differences are taken with a step $j h$, ( $h$ is the pixel width and $j=1,2, \ldots$, ) the range of corner-rounding effects resulting from finite differencing will be $(-j, j)$, where the changes in tangent orientation $\phi$ at each points $x_{-k}$ and $x_{k}$ $(k=0,1, . . j-1)$ are $[8]$ :

$$
\begin{array}{rlr}
\operatorname{tg} \phi_{-k} & =\frac{(j-k)(p-q)}{(j-k)(1+p q)+k\left(1+q^{2}\right)} & \\
& =\frac{p-q}{1+p q+\frac{k}{j-k}\left(1+q^{2}\right)} & \\
\operatorname{tg} \phi_{k} & =\frac{(j-k)(p-q)}{(j-k)(1+p q)+k\left(1+p^{2}\right)} & \\
& =\frac{p-q}{1+p q+\frac{k}{j-k}\left(1+p^{2}\right)} &
\end{array}
$$

Thus a corner is no longer an isolated high curvature point, but one of a group of high curvature points, and the range of those high curvature points depends on the finite difference step as shown in Figure A1 in Appendix A.

To limit the range of corner-rounding effects resulting from finite differencing, a small step such as $j h=2 h$ is adopted since it introduces less error in the computing of tangent orientation to arclength (cf. Figure A3). Meanwhile instead of using a high curvature threshold to filter individual corners, a low curvature threshold is used to filter a range of high curvature points containing the corner that is then located at the point where the curvature magnitude reaches the maximum in this range.

This treatment also tends to extract as long an elliptic arc as possible in segmentation, which is essential for accurate ellipse fitting, as shown in Figure 3.

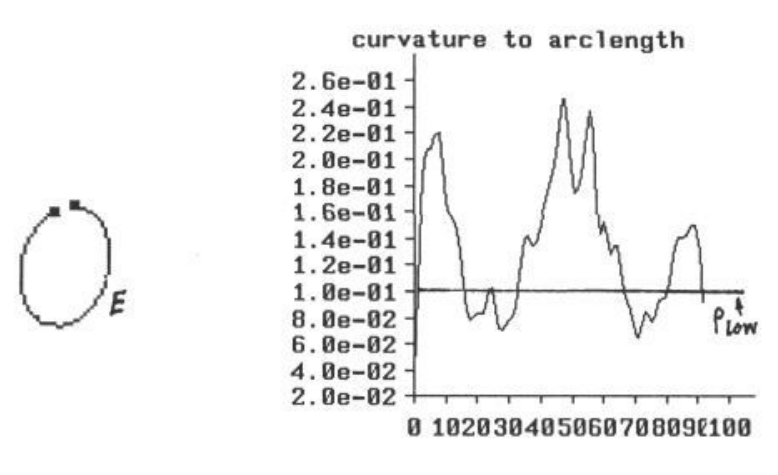

Figure 3. An elliptic curve $E$ is extracted as a whole by a strategy that first searching for the maximum curvature point in an interval determined by an appropriate low curvature threshold $\rho_{\text {low }}$ then extracting a shape descriptor and merging arcs based on their global behaviour.

Another reason to have a local behaviour analysis is that tangent joins show drift effects due to data smoothing and finite differencing [8]. Theoretically, tangent join $x_{0}$ can be detected using zero curvature. However, in practice it is not as simple as being supposed to be. As an example, consider a curve illustrated in Figure 4, where a circular arc $p(x)$ of curvature $\rho$ is tangent to a horizontal line $q(x)$ at $x_{0}$. As a zero curvature point, the tangent join may drift rightwards from the exact position $x_{0}$ after smoothing or finite differencing. This drift depends on smoothing scale and finite difference step. Some analytic results are given here and simulated results are shown in Figure A2 in Appendix A.

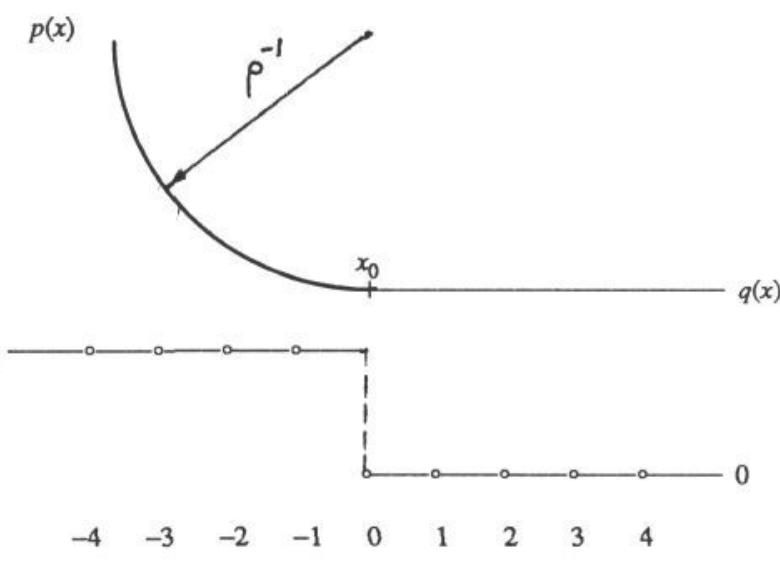

Figure 4. A curve $f(x)$ and its theoretical curvature distribution. $f(x)$ is consist of a circular segment $p(x)$ tangent to a horizontal line segment $q(x)$ at $x_{0}$.

When the smoothing mask applied to curves is:

\begin{tabular}{|l|l|l|}
$\frac{1}{2 a+b} \times \quad a$ & $b$ & $a$ \\
\hline
\end{tabular}

with $\mathrm{n}$ iterations of smoothing, curvature value at $x_{n-1}$ and $x_{n}$ will be:

$$
\begin{aligned}
C_{n-1}^{(n)} & =\frac{a}{2 a+b} C_{n-2}^{(n-1)}=\cdots=\left(\frac{a}{2 a+b}\right)^{n} C_{-1}^{(0)} \\
& =\left(\frac{a}{2 a+b}\right)^{n} \rho
\end{aligned}
$$




$$
C_{n}^{(n)}=0
$$

So the tangent join may drift from $x_{0}$ to $x_{n}$ as long as the curvature at $x_{n-1}$ and the given zero curvature threshold $\rho_{\text {zero }}$ satisfies:

$$
\rho_{\text {zero }} \leq\left|C_{n-1}^{(n)}\right|=\left(\frac{a}{2 a+b}\right)^{n}|\rho|
$$

Hence, it is likely that there will be little zero-drift if the following inequality is satisfied:

$$
\rho_{\text {zero }} \geq \frac{a}{2 a+b}|\rho|
$$

As for the effects resulted from finite differencing, when the difference step takes $2 h$, the curvature at $x_{1}$ is:

$$
\left|C_{1}\right|=\frac{\left|\ddot{f}_{1}\right|}{\left(1+\dot{f}_{1}^{2}\right)^{3 / 2}}=\frac{\frac{1}{8}|\rho|}{\left(1+\frac{1}{16} h^{2} \rho^{2}\right)^{3 / 2}}<\frac{1}{8}|\rho|
$$

the tangent join may drift from $x_{0}$ to $x_{2}$ as long as the curvature magnitudes at $x_{1}, x_{2}$ satisfy:

$$
\begin{gathered}
\rho_{\text {zero }}<\left|C_{1}\right|<\frac{1}{8}|\rho| \\
C_{2}=0
\end{gathered}
$$

Hence, to guard against drift effects, the zero curvature threshold should satisfy:

$$
\rho_{\text {zero }} \geq \frac{1}{8}|\rho|
$$

Figure 5 shows an elliptical arc with two tangent lines, the location of the tangent joins $A$ and $B$ and the zero curvature threshold in the curvature vs arclength map. The another part of the same ellipse and a small ellipse are also shown on the image as verification.
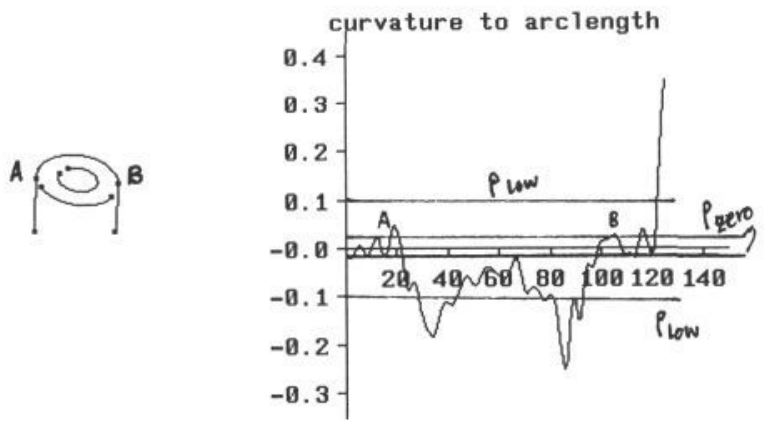

Figure 5. Tangent joins $\mathrm{A}$ and $\mathrm{B}$ are located using the zero threshold illustrated in the map of curvature vs. arclength.

\section{Global behaviour of planar curves}

Although curvature is a powerful tool in describing the local behaviour of curves at individual points, espe- cially for events accompanied by large and abrupt shape changes, it is less able to describe the behaviour of curves at events accompanied by small and gradual shape changes, e.g., the following two cases:

1) A long running curve of fairly low curvature, of which a short segment is nearly a straight line.

2) The line edge(s) of a corner whose curvature is "corrupted" by high curvature due to cornerrounding effects.

Describing behaviour of curves at these events requires an analysis of the global behaviour of the curve segment. Global behaviour will be described in terms of the quantity $S_{0}^{n}$, mean polygonal area along the curve,

$$
S_{0}^{n}=\frac{1}{2 n}\left[\left|\begin{array}{ll}
x_{0} & x_{1} \\
y_{0} & y_{1}
\end{array}\right|+\left|\begin{array}{ll}
x_{1} & x_{2} \\
y_{1} & y_{2}
\end{array}\right|+\cdots+\left|\begin{array}{cc}
x_{n-1} & x_{n} \\
y_{n-1} & y_{n}
\end{array}\right|+\left|\begin{array}{cc}
x_{n} & x_{0} \\
y_{n} & y_{0}
\end{array}\right|\right)
$$

As illustrated in Figure $6, S_{0}^{n}$ is the algebraic sum of areas enclosed by the curve and its chord weighted by $n^{-1}$ in favour of arcs, where $\mathrm{n}$ is the number of points of the arc (cf. Appendix B).

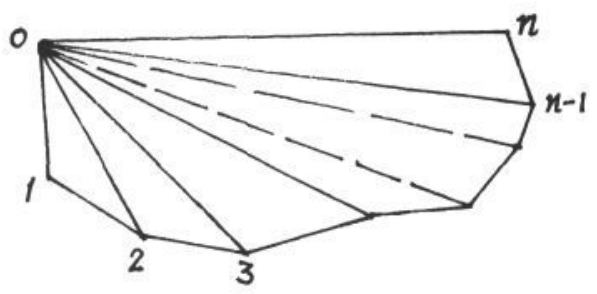

Figure 6. The polygonal area of an open curve $f(x)$.

Using mean polygonal area, long running, fairly low curvature arcs can be distinguished from straight lines owing to the monotonic increase in the polygonal area of these arcs as shown in Figure 7.

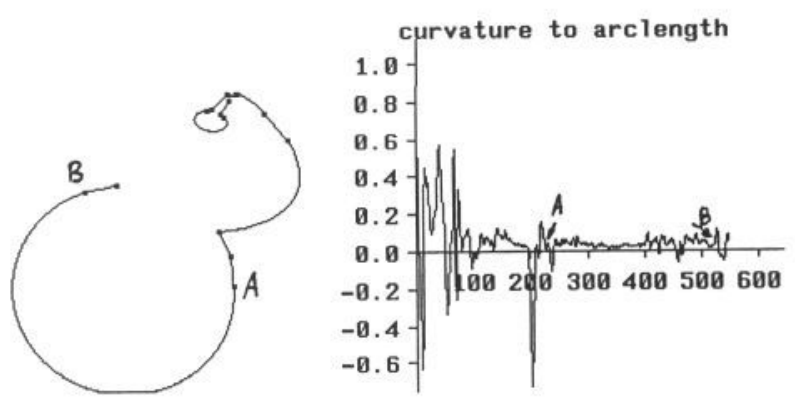

Figure 7. Extraction of long running, low curvature arcs, such as $A^{\cap} B$ using mean polygonal area

As for the line edges of comers types $0 / 0,0 /-$ or $0 /+$, the mean polygonal areas of line edges are nearly zero. So they can be distinguished from the curved edges of other types due to the independence of this quantity to the calculation of orientation change $\phi$ or curvature $\rho$. 
5. Duda, R. D., and Hart, P. E. Pattern Classification and Scene Analysis, New York: Wiley, 1973.

6. Pavlidis, T. "Segmentation of pictures and maps through functional approximation," Computer Graphics, Image processing 1, 360-372. 1972.

7. Asada, H. and Brady, M. "The Curvature Primal Sketch," Technical Report AIM-758, MIT, AI Lab., 1984.

8. Cai, L. D. "Zero-drift effects in location of tangent joins of planar curves," DAI Working Report No. , Department of A.I., University of Edinburgh, 1989.

9. Cai, L. D. "Spline Smoothing: A Special Case of Diffusion Smoothing," Proc. of the 5th AVC conference, Reading, UK, July, 1989, pp. 273-276.

10. Porrill, J. "Fitting Ellipses and Predicating Confidence Envelopes using a Bias Corrected Kalman Filter", Proc. of the 5th AVC conference, Reading, UK, July, 1989, pp. 175-180.

\section{Appendix A. Corner rounding and tangent join drift effects}

The following figures show the corner rounding effects resulting from finite differencing and the tangent join drift effects resulting from both data smoothig and finite differencing.

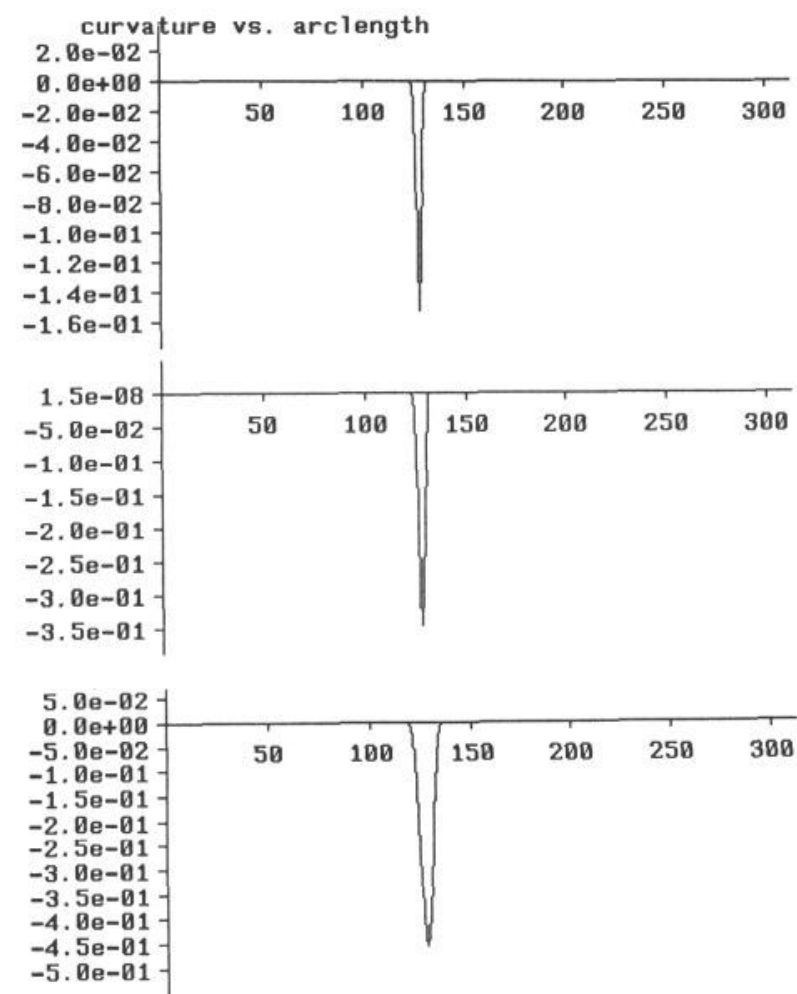

Figure A1. The range of high curvature points near the comer $x_{0}=100$ expands when the finite difference step $j h$ increases $\left(\phi_{0}=30^{\circ}, j=1,3,8\right)$.
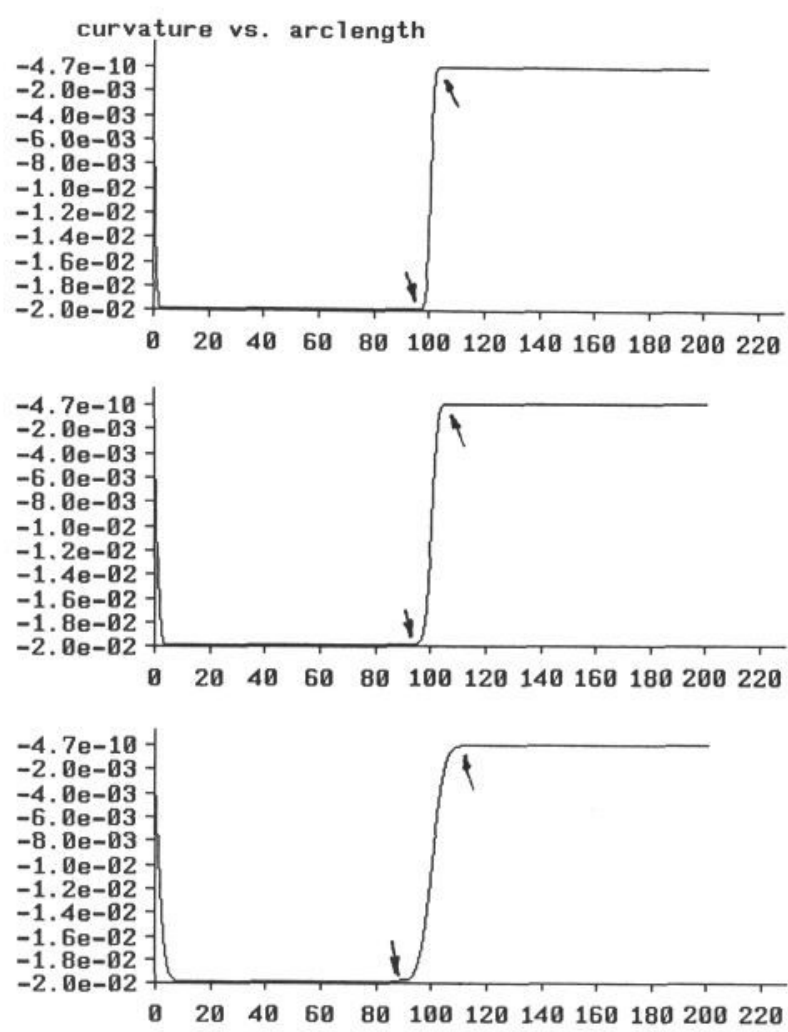

Figure A2. The position of zero curvature point drifts rightwards gradually when the smoothing scale increases. $(j=1,2,4)$.
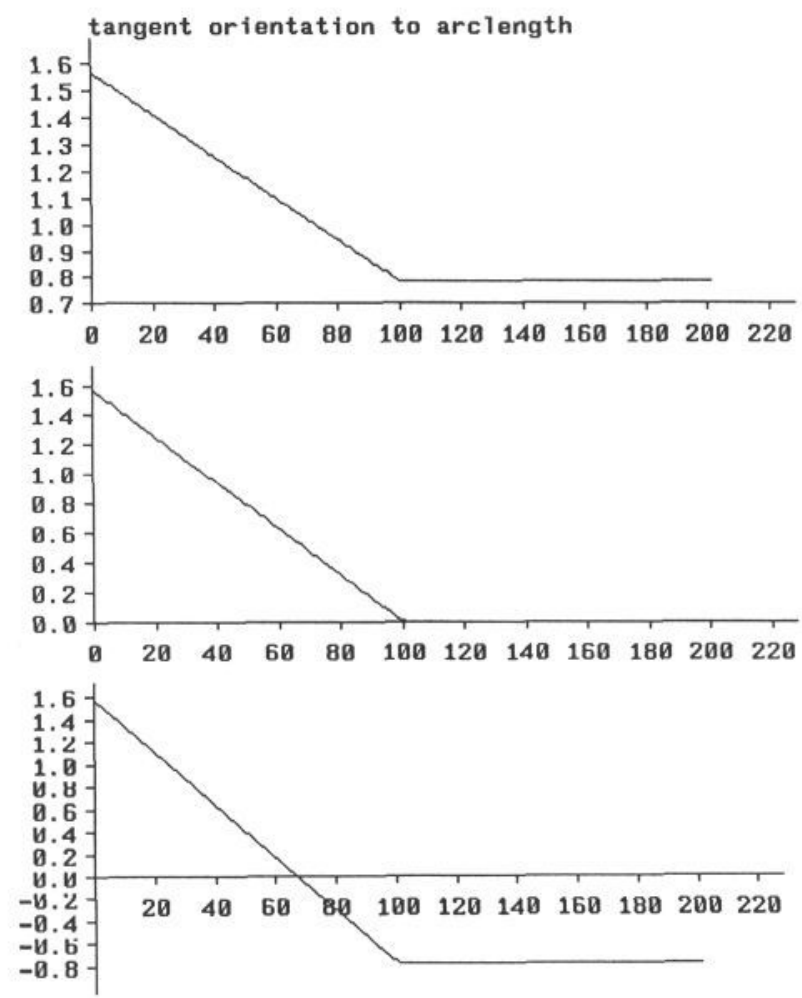

Figure A3. Computing the tangent orientation cross the tangent join $x_{0}=128$ using different finite difference steps. Less error is introduced in the result when the finite difference step is $j h=2 h$. 

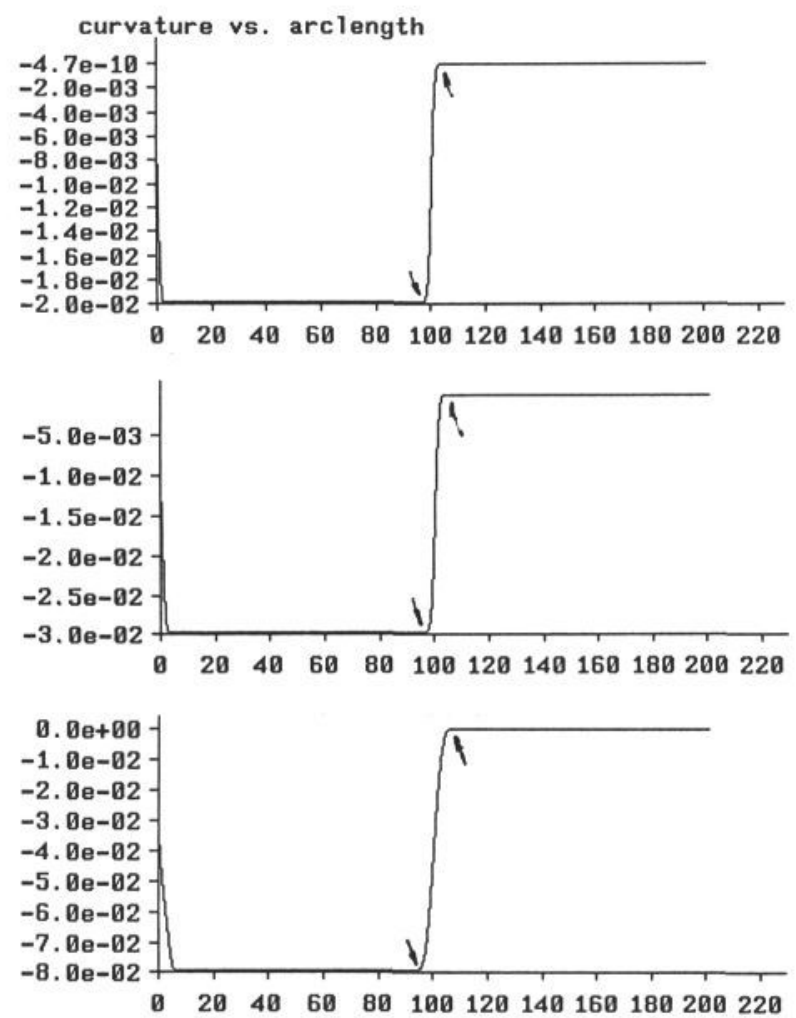

Figure A4. The position of zero curvature point drifts rightwards gradually when the finite difference step increases. $\left(x_{0}=100, j=1,3,8\right)$.

\section{Appendix B. Mean polygonal area of 2-D curves}

Definition 1: Given an open curve $f(t), t \in[0, n]$ in polygonal form: $f_{0}^{n}=\overline{f_{0} f_{1}}+\overline{f_{1} f_{2}}+\cdots+\overline{f_{n-1} f_{n}}$. The chord of the curve over the interval $[0, n]$ is $f_{0} f_{n}$, and the closed polygon formed by this curve is $P_{0}^{n}=f_{0}^{n}+\overline{f_{n} f_{0}}$.

Definition 2: Given an open curve $f(t), t \in[0, n]$ in polygonal form, the polygonal area of the curve, $S_{0}^{n}$, is the area of its polygon $P_{0}^{n}$ :

$$
\begin{aligned}
S_{0}^{n} & =S_{\Delta 0,1,2}+S_{\Delta 0,2,3}+S_{\Delta 0,3,4}+\cdots+S_{\Delta 0, n-1, n} \\
& =\frac{1}{2}\left(\left|\begin{array}{ll}
x_{0} & x_{1} \\
y_{0} & y_{1}
\end{array}\right|+\left|\begin{array}{ll}
x_{1} & x_{2} \\
y_{1} & y_{2}
\end{array}\right|+\cdots+\left|\begin{array}{ll}
x_{n-1} & x_{n} \\
y_{n-1} & y_{n}
\end{array}\right|+\left|\begin{array}{ll}
x_{n} & x_{0} \\
y_{n} & y_{0}
\end{array}\right|\right)
\end{aligned}
$$

Where $S_{\triangle A, B, C}$ is the area of triangle $\triangle A B C$, its sign being positive when the loop A-B-C-A is anti-clockwise; negative when the loop A-B-C-A is clockwise, and, obviously, zero when A, B and C are collinear. Hence, when going along the chord $\overline{f_{0} f_{n}}$, regions on the left side of the chord have negative areas whereas regions on the right side have positive areas.

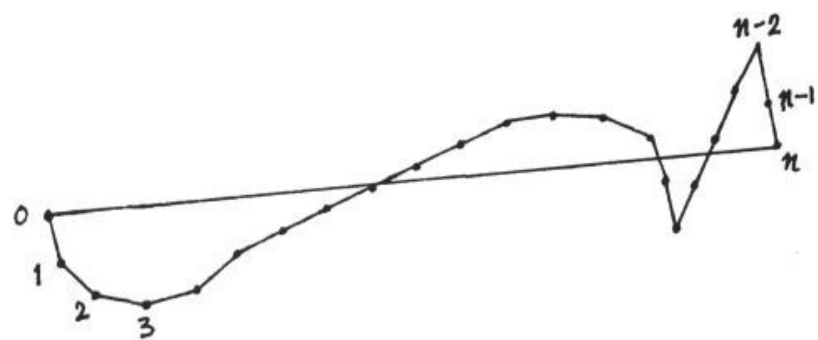

Figure B1. The polygonal area of an open curve $f(x)$.

The polygonal area $S_{0}^{n}$ is actually the algebraic sum of areas of all regions enclosed by curve $f_{0}^{n}$ and its chord $\overline{f_{0} f_{n}}$. For example, the polygonal area $S_{0}^{3}$ illustrated in Figure 9 is

$$
\begin{aligned}
S_{0}^{3} & =S_{\Delta 0,1,2}+S_{\Delta 0,2,3}=S_{\Delta 0,1, c}+S_{\Delta 0, c, 2}+S_{\Delta 0,2, c}+S_{\Delta c, 2,3} \\
& =S_{\Delta 0,1, c}+S_{\Delta c, 2,3}=\left|S_{\Delta 0,1, c}\right|-\left|S_{\Delta c, 2,3}\right|
\end{aligned}
$$

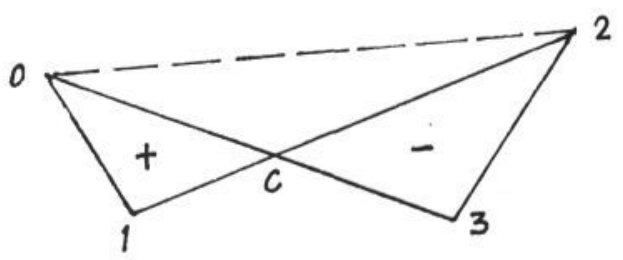

Figure B2. The polygonal area $S_{0}^{3}$ as the algebraic sum of the areas on both sides along the chord $\overline{f_{0} f_{3}}$, where an area on the left side are taken as negative and on the right side as positive.

Definition 3: The mean polygonal area of $f(t), t \in[0, n]$, is the magnitude of the polygonal area divided by $n$.

$$
\overline{S_{0}^{n}}=\frac{1}{n}\left|S_{0}^{n}\right|
$$

From the above definitions, it can be seen that

- The polygonal area and its mean are quantities suitable to sequential low cost computation.

- The polygonal area is zero for a straight line.

- The polygonal area changes monotonically along a convex or concave curve.

- The mean polygonal area is robust to small perturbations of the curve segments.

- The mean polygonal area is independent of the calculation of curvature, thus it is not influenced by corner-rounding effects.

Hence, a threshold can be set up using the mean polygonal area, which will filter long running, low curvature arcs and distinguish line edges of a comer even though false information about the curvature is given there due to the corner-rounding effects. 


\section{Segmentation of planar curves}

Using the above analysis of the behaviour of corners and tangent joins, the curve segmentation algorithm consists of four stages:

1) Reduce data noise with B-spline smoothing [9] to planar curves.

2) Partition the curve into several pieces with a high/low 2-level curvature classifier to identify the ranges of corner candidates.

3) Refine the above result with a high/low/zero 3-level curvature classifier to separate low curvature arcs from zero curvature straight lines.

4) Process the refined segmentation result with a knot/straight/convex/concave shape classifier to get a set of semantic descriptors of all curve segments. Meanwhile, merge adjacent high and low curvature arcs into one, if possible.

As an example, Figure 8 shows the result of segmentation of the Canny edges of a group of widget parts.
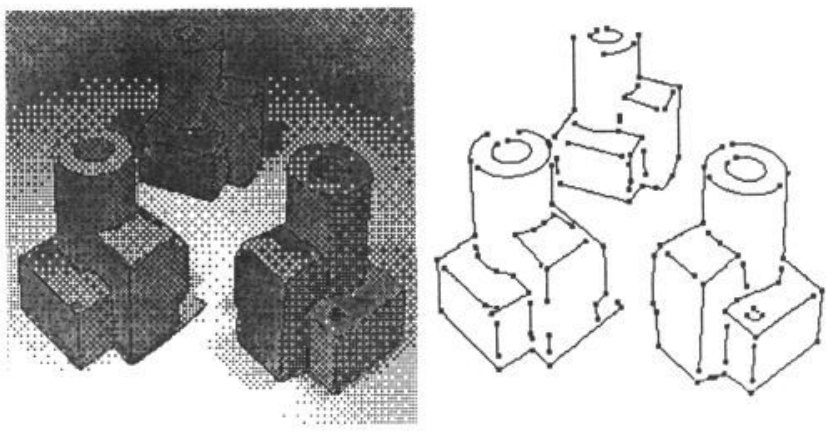

Figure 8. Segmentation of the contours of widget parts.

\section{Symbolic representation of planar curves}

Curve segmentation provides a set of critical knots and shape descriptors of each segment. A symbolic representation can be made on this basis.

So far curve fitting has not been involved in the partitioning stage. The curve is now piecewisely approximated either by a straight line or a quadric curve. In this way a reconstructed version of the segmented curve can be produced by joining the partitioning knots along the curve by the linear or quadric approximations.

Figure 9 shows the reconstruction of the contour of a pair of scissors after segmentation and approximation.

In the examples illustrated in this paper straight sections are approximated by joining their endpoints and conic arcs by finding the conic through the endpoints and three intermediate points. In a future paper we will describe other fitting methods based on the optimal ellipse fitting method [10].
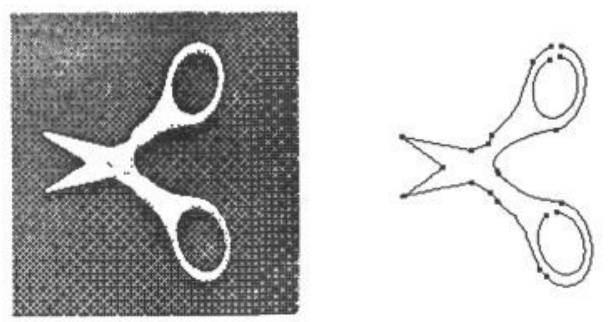

Figure 9. Contour of a pair of scissors approximated piecewisely by linear and quadric curves.

Although the segmentation method proposed in this paper does not always succeed in extracting an elliptic curve as a whole, experimental results so far have shown it does succeed in many cases with the same choice of low and zero curvature thresholds.

\section{Summary}

The detection and location of curvature discontinuities is crucial for the segmentation and representation of planar curves. In this paper, planar curve segmentation is achieved based on an analysis of the behaviour of curvature and mean polygonal area, both being intrinsic properties of planar curves. The corner-rounding effects and the tangent join drift effects resulting from data smoothing and finite differencing are limited by setting up appropriate low and zero curvature thresholds. A rough segmentation of curves are produced by a high/low 2-level curvature classifier, then refined by a high/low/zero 3-level curvature identifier and a knot/straight/convex/concave shape identifier so as to obtain a set of critical knots and semantic shape descriptors for all segments. Afterwards a linear or quadric curve approximation is applied to each segment to produce a symbolic representation.

\section{References}

1. Porrill J., Pollard S. B., Pridmore T. P., Bowen J. B., Mayhew J. E. W. \& Frisby, J. P. "TINA: The Sheffield Vision System," Proceedings of the Ninth International Joint Conference on Artificial Intelligence, Milan, pp. 1138-1144, 1987.

2. Pollard, S. B., Porrill J. and Meyhew, J.E.W., "Using 2D constraints in the 3D interpretation of stereo data," Proceeding of the BMVC conference, Oxford, UK, September, 1990.

3. Pridmore, A. P., Porrill, J., and Mayhew, J. E. W. "Segmentation and description of binocularly viewed contours", Image and Vision Computing, Vol. 5, No. 2, May 1987, pp. 132-138.

4. Cai, L. D. "Approximating A surface up to curvature signs using depth data alone," Proc. of the SPIE/SPSE Symposium on Curves and Surfaces in Computer Vision and Graphics, Santa Clara, California, February 11-16, 1990. 